\title{
Prevalencia de factores de riesgo para infección por Klebsiella pneumoniae re- sistente a carbapenémicos en adultos en un hospital de cuarto nivel, Bogotá
}

\author{
Carlos Saavedra 1 , Viviana López ${ }^{2}$, Paola Linares ${ }^{3}$, Pablo Romero ${ }^{4}$, Carlos Solórzano ${ }^{5}$, Javier Mora ${ }^{6}$, Alan Ovalle.
}

1. Médico Internista. Infectólogo. Epidemiólogo Clínico. Hospital Universitario Nacional de Colombia.

2. Médico Internista. Hospital Universitario Clínica San Rafael.

3. Médico Internista. Hospital Santa Clara.

4. Médico General. Epidemiólogo

5. Médico Internista. Hospital Universitario Clínica San Rafael.

6. Médico Internista. Epidemiólogo. Hospital Universitario Clinica San Rafael.

7. Médico Internista. Hospital Universitario Clínica San Rafael.

Autor de correspondencia:

Viviana López,

vivilopez0321@gmail.com

Saavedra C, López V, Linares P, $\quad$ Romero P, Solórzano C, Mora J, Ovalle A. Prevalencia de factores de riesgo para infección por Klebsiella pneumoniae resistente a carbapenemicos en adultos en un hospital de cuarto nivel, Bogotá D.C. Revista Cuarzo 2018:24 (2) 13-19.

Recibido: 9 de agosto de 2018 Aceptado: 20 de octubre de 2018 Publicado: 30 de diciembre de 2018
Resumen

Introducción: Las infecciones por gram negativos adquiridas en el hospital son una causa importante de muerte, Klebsiella pneumoniae es una de las bacterias más significativas, está además relacionada con la aparición de nuevos mecanismos de resistencia antibiótica. Los carbapenémicos han permitido combatir ciertas de estas cepas resistentes, sin embargo, la aparición de algunas con resistencia a estos antibióticos representa un reto terapéutico. A partir de este contexto clínico se planteó establecer la prevalencia de factores de riesgo en pacientes adultos con aislamientos de Klebsiella pneumoniae resistente a carbapenémicos.

Material y métodos: Se realizó un estudio descriptivo retrospectivo en el Hospital Universitario Clínica San Rafael de Bogotá (Colombia).

- Selección de pacientes: A partir de 295 historias clínicas revisadas de pacientes hospitalizados con aislamiento positivo para Klebsiella pneumoniae con perfil de resistencia a carbapenémicos confirmada en el periodo enero 2013 a diciembre 2015, se obtuvieron 84 pacientes que cumplían todos los criterios de inclusión.

- Mediciones: Se tuvieron en cuenta variables demográficas como sexo y edad, así como las diferentes condiciones probablemente asociadas a la condición infecciosa.

- Métodos analíticos: A las variables cualitativas se les calcularon frecuencias absolutas y relativas, y a las variables cuantitativas medidas de tendencia central y de dispersión.

Resultados: De 84 pacientes, el 55\% correspondía a sexo femenino, tenían una edad media de 66 años. El aislamiento microbiológico en orina fue del $30.4 \%$, en sangre $24.3 \%$, liquido peritoneal $20.7 \%$ y secreción traqueal $12.1 \%$. El 91,6\% de la población tenía hospitalización en los últimos 3 meses, $86.5 \%$ recibió tratamiento antibiótico durante la hospitalización, de estos el 59.1\% fue Meropenem, el 26,2\% fue Ampicilina Sulbactam, el 23,9\% fue Vancomicina, el 18,3\% fue Piperacilina Tazobactam. El 54,7\% se sometió a algún procedimiento quirúrgico 3 meses antes del aislamiento, de los cuales el $47.8 \%$ correspondía a cirugías abdominales y el 13,1\% a cirugía de tórax, luego aparecían cirugía ortopédica, en sistema nervioso central y ginecológica. El 70\% de los casos tenían antecedente de implante de dispositivos intravasculares, $45,2 \%$ exposición a ventilación mecánica, $24 \%$ uso de nutrición parenteral.

Conclusiones: Este estudio establece las bases para proponer nuevos estudios que planteen estrategias de prevención con el objetivo de lograr disminuir las probabilidades de adquirir esta infección multirresistente.

Palabras clave: Klebsiella pneumoniae, antibacterianos, drogo resistencia, factores de riesgo, carbapenémicos 


\title{
Prevalence of risk factor for Klebsiella pneumoniae carbapenemic-resistant in- fection in adults in a fourth level hospital, Bogota.
}

\begin{abstract}
Introduction: Gram-negative infections acquired in the hospital are an important cause of death, Klebsiella pneumoniae is one of the most significant bacteria, it is also related to the appearance of new mechanisms of antibiotic resistance. The carbapenems have allowed to fight certain of these resistant strains, however, the appearance of some with resistance to these antibiotics represents a therapeutic challenge. From this clinical context, it was proposed this research, to establish the prevalence of risk factors in adult patients with isolates of Klebsiella pneumoniae resistant to carbapenems.
\end{abstract}

Material and methods: A retrospective descriptive study was carried out in the San Rafael Clinical University Hospital of Bogotá (Colombia).

- Selection of patients: From 295 reviewed clinical records of patients hospitalized with positive isolation for Klebsiella pneumoniae with carbapenemic resistance profile confirmed, in the period January 2013 to December 2015, 84 patients were obtained who fulfilled all the inclusion criteria.

- Measurements: Demographic variables such as sex and age were taken into account, as well as the different conditions probably associated with the infectious condition.

- Analytical methods: Qualitative variables were calculated absolute and relative frequencies, and quantitative variables measures of central tendency and dispersion were calculated.

Results: Of 84 patients, $55 \%$ corresponded to females, had a mean age of 66 years. The microbiological isolation in urine was $30.4 \%$, in blood $24.3 \%$, peritoneal fluid $20.7 \%$ and tracheal secretion $12.1 \% .91 .6 \%$ of the population had hospitalization in the last 3 months, $86.5 \%$ received antibiotic treatment during hospitalization, of these 59.1\% was Meropenem, 26.2\% was Ampicillin Sulbactam, 23.9\% was Vancomycin, 18.3\% was Piperacillin Tazobactam. 54.7\% underwent some surgical procedure 3 months before the isolation, of which $47.8 \%$ corresponded to abdominal surgeries and $13.1 \%$ to thoracic surgery, then they appeared orthopedic surgery, in central nervous system and gynecological. $70 \%$ of the cases had antecedents of implantation of intravascular devices, $45.2 \%$ exposure to mechanical ventilation, $24 \%$ use of parenteral nutrition.

Conclusions: This research establishes the bases to propose new researches that propose prevention strategies with the objective of reducing the chances of acquiring this multiresistant infection.

Keywords: Klebsiella pneumoniae, anti-bacterial agents, drug resistance, risk factors, carbapenems

\section{Introducción}

Las infecciones adquiridas en el hospital tales como neumonías, infecciones urinarias y bacteriemias causadas por gram negativos son una causa importante de muerte siendo esta la sexta en Europa y Estados Unidos, aunque hay varias bacterias implicadas, Klebsiella pneumoniae es una de ellas, ahora además relacionada con la aparición de nuevos mecanismos de resistencia antibiótica, convirtiéndose en un problema de salud pública (1).

El uso de carbapenémicos ha permitido combatir ciertas cepas resistentes, sin embargo la aparición de nuevas cepas de Klebsiella con resistencia a estos antibióticos representa un reto terapéutico (1), estas cepas se han relacionado con infecciones sistémicas en el contexto intrahospitalario y de unidades de cuidado intensivo (UCI), generando altas tasas de morbilidad, mortalidad, y aumentando los costos en salud (2).
La resistencia a carbapenémicos por Klebsiella pneumoniae ha crecido rápidamente desde el 2001, se ha reportado en muchas ciudades, siendo ahora endémica en el noreste de Estados Unidos, Puerto Rico, Grecia, algunas provincias de China, e Israel (3). El primer aislamiento en Suramérica fue realizado en Colombia (4), donde además se encontró resistencia a carbapenémicos en otras enterobacterias, de forma similar se hicieron aislamientos en Brasil y Argentina.

En el Hospital San Ignacio junto con la Universidad del Rosario, realizó un estudio no publicado de casos y controles para establecer los factores de riesgo para infección y colonización por Klebsiella pneumoniae resistente a carbapenémicos, si bien los factores de riesgo no están completamente claros, a partir de los ya descritos se han tomado medidas de intervención importantes que han permitido el control de brotes de este tipo de infecciones. 
Las enterobacterias Escherichia coli y Klebsiella pneumoniae son las causales más frecuentes de infecciones bacterianas adquiridas en el hospital y la comunidad, la presencia de resistencia a carbapenémicos en estas bacterias envuelve la necesidad de control y detección como punto crítico para un adecuado manejo de los pacientes desarrollando adecuadas estrategias de prevención (5), no hacerlo trae implicaciones clínicas y epidemiológicas para la sociedad. En estudios previos se ha evaluado la mortalidad en pacientes con Klebsiella resistente a carbapenémicos, resistente a no carbapenémicos y con otras enterobacterias, encontrando esta infección como predictor claro de mortalidad $(7,8)$.

Se ha descrito que la sepsis severa y choque séptico por gram negativos expuestos previamente a antibióticos, aumenta la mortalidad en $51,3 \%$ vs $34 \%$ respecto a quienes no los han recibido, además en este contexto existe un aumento de 5-7 días en estancia hospitalaria, así como en costos en salud, el uso previo de antibióticos como Cefepime, Ciprofloxacina, Ceftriaxona, e Imipenem, han sido los más asociados a este tipo de infecciones, la explicación está dada por aumento de los mecanismos de resistencia bacteriana.

Se ha notado que los costos vienen en aumento al pasar de los años, así lo registró el Instituto Nacional de Enfermedades Infecciosas y Alérgicas (NIAID) (9), además se describe una franca diferencia en aquellos pacientes con infección adquirida en comunidad e infección asociada al cuidado de la salud, con resistencia bacteriana no solo en costos, sino también en estancia hospitalaria, y probabilidad de muerte en relación a aquellos con susceptibilidad a antibióticos (10).

No hay datos en Colombia sobre costos institucionales al respecto, sin embargo, los datos universales son contundentes, a partir de lo anterior nace la importancia de conocer cuáles son los factores de riesgo más prevalentes para adquirir Klebsiella pneumoniae resistente a carbapenémicos, y de esta manera poder establecer estrategias de prevención, algunos de estos factores ya están claramente identificados y otros han tenido resultados variables en los diferentes estudios.

Se ha encontrado que algunos factores que se asociaban a esta bacteria eran la estancia en la UCI, severidad de la enfermedad calculada por APACHE II, uso de catéter venoso central, uso de sonda vesical, y uso previo de antibióticos, entre otros (11). También se ha encontrado asociación con enfermedad severa, así como uso previo de antibióticos tipo Fluoroquinolonas o Cefalosporinas de espectro extendido, confirmándose en todos estos casos aumento en la mortalidad de este tipo de pacientes (8).

En nuestra institución se han encontrado casos de infección por este tipo de bacteria, lo cual nos permitió realizar este estudio, el objetivo fue establecer la prevalencia de factores de riesgo en pacientes adultos con aislamientos de Klebsiella pneumoniae resistente a carbapenémicos, teniendo en cuenta las características de la población, y haciendo énfasis especial a la exposición previa a los antibióticos y exposición previa a cirugía, de esta manera detectar los puntos clave para establecer estrategias y recomendaciones para nuestra y otras instituciones de nuestro medio, generando impacto en la morbilidad y mortalidad de estos pacientes.

\section{Material y Métodos}

- Tipo y diseño de estudio: Se realizó un estudio descriptivo retrospectivo.

- Población en estudio: Pacientes hospitalizados en el periodo enero 2013 a diciembre 2015 en el Hospital Universitario Clínica San Rafael, adultos ( $>18$ años), a quienes se les aisló Klebsiella pneumoniae con perfil de resistencia a carbapenémicos confirmada. Se tuvieron en cuenta los siguientes criterios de inclusión y exclusión:

Criterios de inclusión: Pacientes con edad $>18$ años, con aislamiento en cualquier muestra microbiológica Klebsiella pneumoniae con perfil de resistencia a carbapenémicos confirmada (Meropenem y Ertapenem), independiente de la realización del test de Hodge modificado, test de ácido borónico y/o test de EDTA, en el contexto de "infección". [Infección: Todo aislamiento positivo en cualquier muestra microbiológica que genere condición de enfermedad, dado por respuesta inmunológica y daño estructural de un hospedero, causada por un microorganismo patógeno]

Criterios de exclusión: Aislamientos que correspondan a colonización o contaminación [Colonización: Todo aislamiento positivo en cualquier muestra microbiológica sin que este produzca enfermedad y que se considere un germen que habite en el sitio de recolección de la muestra.

Contaminación: Todo aislamiento positivo que no corresponde a un germen de la flora normal del sitio de recolección, no produce enfermedad y suele estar relacionado a errores en la toma de la muestra], pacientes con el segundo episodio de infección por Klebsiella pneumoniae resistente a carbapenémicos, aquellos con hospitalización previa en los últimos 3 meses en otra institución lo cual podría dificultar obtener los datos y cambiar la epidemiologia de nuestra institución, pacientes con aislamiento simultaneo de otra bacteria con patrón de resistencia que requiera terapia con carbapenémicos.

- Variables en estudio: Se clasificaron de la siguiente manera:

Variables resultado: uso previo de esteroides, uso previo de antibióticos intrahospitalarios, uso previo de antibióticos extra hospitalarios (en casa), tipo de antibióticos previos intra o extra hospitalarios, cirugía previa, tipo de cirugía previa, uso de dispositivos intravasculares, uso de ventilación mecánica, número de días de ventilación mecánica, uso de nutrición parenteral, otros dispositivos invasivos, neutropenia, diálisis, hospitalización previa en estancia general, número de días de hospitalización en estancia general, hospitalización previa en cuidados intensivos, 
número de días de hospitalización en cuidados intensivos, origen del aislamiento.

Variables secundarias: sexo, edad, nivel de comorbilidad (Escala de Charlson) (11).

- Plan de recolección de datos: Previa aprobación por el grupo de investigación y comité de ética médica, la información se recolectó a partir del número de casos informados por la base de datos del laboratorio del Hospital Universitario Clínica San Rafael en el periodo comprendido entre enero de 2013 y diciembre de 2015, esta base de datos está fundamentada en métodos automatizados BD Epicenter (Becton Dicknson) con 99\% de confiabilidad y en donde se informaron los cultivos de cualquier muestra microbiológica positivos para Klebsiella pneumoniae resistentes a carbapenémicos confirmada según los parámetros de MICs (concentración inhibitoria mínima) del CLSI (Clinical and Laboratory Standars Institute) de pacientes que cumplían el rango de edad, fecha de hospitalización, y demás criterios de inclusión en la población en estudio; posteriormente se procedió a la revisión de historias clínicas a través del sistema "HEON (Health online)" al cual tenemos acceso mediante las claves de acceso personal, y el cual permite la visualización de los reportes de laboratorio clínico así como de historias clínicas de los pacientes seleccionados, identificando cada una de las variables establecidas a estudiar, finalmente esta información fue registrada y recopilada en Excel a través de la herramienta Google Drive. No se realizó cálculo de la muestra, dado que fueron revisadas todas las historias clínicas obtenidas (295), recolectándose finalmente un total de 84 pacientes que cumplieron criterios de inclusión y no los de exclusión.

- Plan de análisis: Los datos obtenidos fueron analizados por los investigadores (quienes no padecen de ninguna enfermedad que pueda alterar su análisis y resultados). Las variables cualitativas se ordenaron en frecuencias absolutas, relativas y dis- tribuciones de frecuencias y a las variables cuantitativas se les calculó medidas de tendencia central y de dispersión pertinentes. Los datos almacenados en Excel durante la recolección de datos fueron analizados en el programa SPSS versión 24.

- Consideraciones éticas: Teniendo en cuenta que la información se recolectó a partir de los registros de historias clínicas y no se hizo ninguna intervención sobre los individuos, se consideró una investigación "sin riesgo", se mantuvo la confidencialidad de la información obtenida de los pacientes seleccionados y se archivaron los datos en las bases de datos del grupo de investigación que se encontraban en la red de internet con clave solo conocida por los investigadores principales, no se tabularon los datos personales de los mismos y se hizo una presentación agrupada de los datos lo cual impidió la eventual identificación de los sujetos reales, el estudio no requirió de consentimiento informado y fue subrogado al comité de ética e investigación de la institución, dado que no se intervino directamente al paciente y este no fue afectado físicamente ni se benefició de los resultados como individuo, durante la recolección de información no se identificaron situaciones de mala práctica, o errores en la atención.

\section{Resultados}

Se revisaron 295 historias clínicas de pacientes con presencia de germen multirresistente de acuerdo a perfil de antibiograma confirmado mediante aislamiento en cultivos según informe del laboratorio, se obtuvo una muestra de 84 pacientes que cumplían todos los criterios de inclusión (Figura 1).

Encontramos que el $55 \%$ de nuestra población correspondía a sexo femenino, se encontraban entre las edades de 18 y 94 años con una media de edad de 66 años, el promedio de supervivencia a 5 años valorada según puntaje en la Escala de Charlson fue del $18 \%$ de la población.

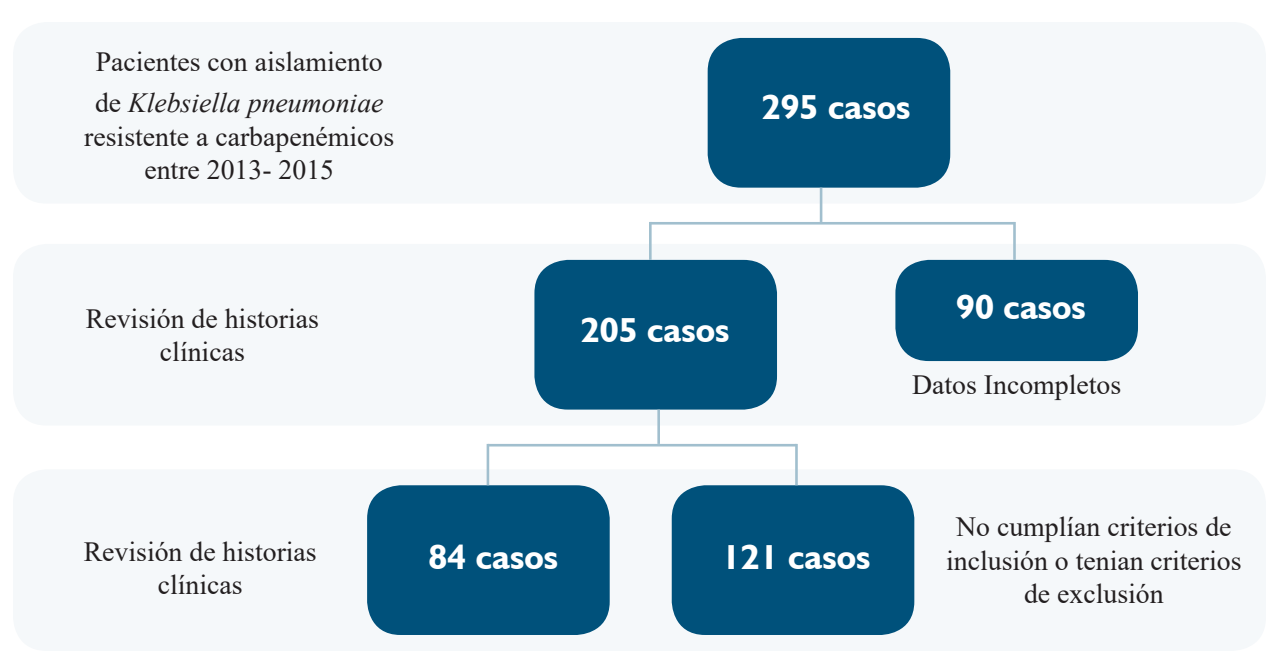

Figura I: Flujograma del proceso de selección de los pacientes incluidos en el estudio. 
El aislamiento microbiológico más frecuente fue en orina con el 30.4\%, los otros aislamientos fueron en sangre 24.3\%, liquido peritoneal $20.7 \%$ y secreción traqueal $12.1 \%$ (Tabla 1 ).

Tabla I: Origen del aislamiento microbiológico

\begin{tabular}{lccc} 
Origen del aislamiento Microbiológico & Número & Porcentaje & IC 95\% \\
\hline Catéter intravascular & 59 & $70.2 \%$ & $59.2-79.7$ \\
Orina & 25 & $30.4 \%$ & $20-40.7$ \\
Sangre & 20 & $24.3 \%$ & $15.1-34.4$ \\
Líquido peritoneal & 17 & $20.7 \%$ & $12.2-30.4$ \\
Secreción traqueal & 10 & $12.1 \%$ & $5.8-20.8$ \\
$\begin{array}{l}\text { Colección o tejido } \\
\text { Catéter de sistema nervioso central o líquido } \\
\text { cefalorraquídeo }\end{array}$ & 5 & $5.9 \%$ & $1.9-13.3$ \\
\end{tabular}

Se encontró que el 91,6\% de la población tenía hospitalización en sala general en los últimos 3 meses y 57\% también tenían estancia en UCI. El 86.5\% (71) de los pacientes recibió tratamiento antibiótico durante la hospitalización y el 7.3\% (6) de forma ambulatoria. De los que recibieron antibiótico, el $59.1 \%$ fue Meropenem, el 26,2\% fue Ampicilina Sulbactam, el 23,9\% fue Vancomicina, el 18,3\% fue Piperacilina Tazobactam, y 12,6\% fueron Cefalosporinas de 4ta Generación (Tabla 2).

Tabla 2: Tipo de antibiótico recibido

\begin{tabular}{|c|c|c|c|}
\hline Tipo de Cirugía & Número & Porcentaje & IC $95 \%$ \\
\hline Meropenem & 42 & $59.1 \%$ & $46.8-70.6$ \\
\hline Ampicilina Sulbactam & 19 & $26.2 \%$ & $16.9-38.5$ \\
\hline Vancomicina & 17 & $23.9 \%$ & $14.6-35.5$ \\
\hline Piperacilina Tazobactam & 13 & $18.3 \%$ & $10.1-29.2$ \\
\hline Cefalosporinas 4ta Generación** & 9 & $12.6 \%$ & $5.9-22.7$ \\
\hline Cefalosporinas 1ra Generación*** & 5 & $7.0 \%$ & $2.3-15.6$ \\
\hline \multicolumn{4}{|c|}{$\begin{array}{l}* \text { De todos los pacientes, solo } 86,5 \% \text { ( } 71) \text { recibieron antibióticos, sin embargo, algunos recibieron varios antibióticos de forma escalonada } \\
\text { previo al aislamiento microbiológico. } \\
* * \text { Cefalosporinas 4ta Generación: Cefepime. } \\
* * * \text { Cefalosporinas 1ra Generación: Cefazolina, Cefalexina. }\end{array}$} \\
\hline
\end{tabular}

El 54,7\% (46) se sometió a algún procedimiento quirúrgico 3 meses antes del aislamiento, de estos, el $47.8 \%$ correspondían a cirugías abdominales, el 13,1\% a cirugía de tórax, y el 10,8\% a cirugía ortopédica, así como del Sistema Nervioso Central, la cirugía ginecológica alcanzó el 4,4\% (Tabla 3).

El 70\% de los casos tenía antecedente de implante de dispositivos intravasculares, la exposición a ventilación mecánica estuvo presente en $45,2 \%$, el uso de nutrición parenteral en $24 \%$, la terapia dialítica en $19 \%$ de los casos, el uso previo de corticoide se encontró en el 17\% de los casos, tan solo el $6 \%$ del total de la población cursó con disminución en el conteo de neutrófilos (Tabla 4).

De los pacientes que recibieron tratamiento con Meropenem, el $33,3 \%$ (16) procedían de aislamiento en sangre y el 20,8\% (10) de orina, para el caso del tratamiento previo con Piperacilina Tazobactam, los aislamientos fueron provenientes de orina en el $42,9 \%$ (6) de los casos seguido por liquido peritoneal en el $28,6 \%$ (4) y de sangre solo en el 7,1\% (1). 
Tabla 3: Tipo de Cirugía recibida.

\begin{tabular}{lccc}
\multicolumn{1}{c}{ Tipo de Cirugía } & Número & Porcentaje & IC 95\% \\
\hline Cirugía abdominal & 22 & $47.8 \%$ & $32.8-63$ \\
Cirugía de tórax & 6 & $13.1 \%$ & $4.9-26$ \\
Otras* & 6 & $13.1 \%$ & $4.9-26$ \\
Cirugía ortopédica & 5 & $10.8 \%$ & $3.6-23$ \\
Cirugía sistema nervioso central & 5 & $10.8 \%$ & $3.6-23$ \\
Cirugía ginecológica & 2 & $4.4 \%$ & $0.05-14.8$ \\
Total & 46 & $100 \%$ &
\end{tabular}

* Otras corresponde a cirugía urológica, biopsia en cualquier sitio anatómico, cirugía de columna, cirugía cardiaca, cirugía en tejidos blandos.

Tabla 4: Condición previa al aislamiento microbiológico.

\begin{tabular}{lccc}
\multicolumn{1}{c}{ Condición previa } & Número & Porcentaje & IC 95\% \\
\hline Hospitalización en sala general & 77 & $91.6 \%$ & $86.3-97.9$ \\
Uso de antibiótico (hospitalario) & 72 & $85 \%$ & $78.3-91.4$ \\
Uso de dispositivo intravascular & 59 & $70 \%$ & $60-81.3$ \\
Procedimiento quirúrgico & 46 & $58.3 \%$ & $48.2-65.1$ \\
Hospitalización en cuidados intensivos & 48 & $57 \%$ & $46-66.1$ \\
Ventilación mecánica & 38 & $45.2 \%$ & $33.9-54$ \\
Uso de nutrición parenteral & 20 & $24 \%$ & $15.5-35.1$ \\
Diálisis & 16 & $19 \%$ & $11.5-29.7$ \\
Uso de corticoide & 14 & $17 \%$ & $9.6-26$ \\
Uso de antibiótico (ambulatorio) & 6 & $7 \%$ & $3.6-14.3$ \\
$\begin{array}{l}\text { Neutropenia }(<500 \text { conteo absoluto de neu- } \\
\text { trófilos) }\end{array}$ & 5 & $6 \%$ & $2.4-10.8$
\end{tabular}

\section{Discusión}

Es bien conocido que Klebsiella pneumoniae hace parte de los aislamientos más importantes de infecciones bacterianas adquiridas en el hospital, y la presencia de resistencia a carbapenémicos en esta bacteria es uno de los factores de mayor preocupación, debido el mal pronóstico esperado en estos casos, así como las dificultades en el tratamiento antibacteriano de los mismos, establecer condiciones de riesgo para adquirir esta bacteria y de esta manera plantear estrategias preventivas cobra gran relevancia en la práctica clínica. El estudio sugiere que la hospitalización previa en estancia general más que en cuidados intensivos, tiene una importancia relevante, lo cual difiere de estudios donde la hospitalización en UCI está más relacionada con esta infección.
En investigaciones previas se ha encontrado que la exposición a antibióticos como Cefepime, Ciprofloxacina, Ceftriaxona, e Imipenem, están asociados con el hallazgo de resistencia a carbapenémicos, en este caso la mayoría de los pacientes habían sido expuestos a antibióticos, pero los que más se describen son Meropenem, Ampicilina Sulbactam, Vancomicina, Piperacilina Tazobactam y en última estancia Cefalosporinas, no se describen Quinolonas ya que existen políticas institucionales de limitación para el uso de estos antibióticos de forma intrahospitalaria.

Respecto al sitio de aislamiento más frecuente y a su vez un probable factor de riesgo potencialmente prevenible fueron los catéteres intravasculares, lo que sugiere que promover estrategias seguras para manejo de estos, así como adecuadas técnicas de asepsia y antisepsia, uso en situaciones de real necesidad, pron- 
to retiro podrían instaurarse para mejorar la tasa de infección por esta bacteria multirresistente, los demás focos de infección fueron orina, sangre, liquido peritoneal, secreción traqueal entre otros.

Otra de las condiciones de riesgo, en cambio no muy descritas en estudios de factores de riesgo para Klebsiella pneumoniae resistente a carbapenémicos, es haber sido sometido a un procedimiento quirúrgico, lo cual se evidenció en la mitad de nuestros pacientes, y de estos, principalmente a cirugía abdominal seguido por cirugía de tórax. Finalmente, el grupo de pacientes con inmunosupresión secundaria a neutropenia (corresponde a los pacientes hospitalizados en nuestra institución por enfermedades hematoncológicas) o uso ambulatorio de antibióticos, no representó una condición de riesgo importante.

Dentro de las limitaciones de este estudio es que este tipo de investigaciones no permite establecer relaciones causales, ni permite estimar el riesgo de un determinado factor, tan solo informamos frecuencias y es cierto que habría enriquecido los resultados calcular razones de prevalencia, para poder afianzar la relación que parece existir entre los factores de riesgo descritos y la enfermedad.

El objetivo de este estudio no era evaluar mortalidad, aunque es bien conocido por resultados de otros autores que la exposición a esta bacteria multirresistente aumenta la mortalidad de forma significativa, para lo que se debe resaltar que la población incluida aquí siendo representación de la población de nuestro hospital es de edad avanzada y con una baja probabilidad de supervivencia a 5 años, por lo que sería difícil plantear a futuro estudios de mortalidad y en caso de realizarlos se deberán establecer criterios de inclusión más selectivos. Finalmente, podemos decir que en nuestro estudio esta bacteria se encontró principalmente en aislamientos de catéteres intravasculares, estuvo presente en quienes habían tenido alguna estancia hospitalaria en UCI o estancia general, un gran porcentaje de pacientes recibió antibiótico previo al aislamiento y el que más se destacó fue el Meropenem como representante de los carbapenémicos, de los llevados a cirugía, fue la intervención abdominal la más frecuente.

Con estos resultados podemos recomendar la promoción de estrategias de prevención institucionales en aquellos factores de riesgo detectados, y promover líneas de investigación en búsqueda de la confirmación de asociación con las condiciones anotadas, con un enfoque especial a la exposición previa de hospitalización, antibióticos y procedimientos quirúrgicos como cirugías abdominales.

\section{Agradecimientos}

A los Dres. Edwin Silva y Carlos Saavedra, por cumplir un papel muy importante en el planteamiento y el desarrollo de este estudio, agradecemos su entrega en el manejo de sus pacientes y entusiasmo por la disminución en la resistencia bacteriana de nuestro hospital.
Al Dr. Aníbal Teherán por sus correcciones durante la realización del anteproyecto de este estudio.

El estudio se realizó con recursos económicos propios de los investigadores y ninguno de ellos declaró conflicto de intereses.

\section{Referencias}

1. Spellberg B, Guidos R, Gilbert D, Bradley J, Boucher HW, Scheld WM, et al. The epidemic of antibiotic-resistant infections: a call to action for the medical community from the Infectious Diseases Society of America. Clin Infect Dis. 2008 Jan 15;46(2):155-64.

2. Patel G, Huprikar S, Factor SH, Jenkins SG, Calfee DP. Outcomes of carbapenem-resistant Klebsiella pneumoniae infection and the impact of antimicrobial and adjunctive therapies. Infect Control Hosp Epidemiol. 2008 Dec;29(12):1099-106.

3. Chen LF, Anderson DJ, Paterson DL. Overview of the epidemiology and the threat of Klebsiella pneumoniaecarbapenemases (KPC) resistance. Infect Drug Resist. 2012;5:133-41.

4. Villegas MV, Lolans K, Correa A, Suarez CJ, Lopez JA, Vallejo M, et al. First detection of the plasmid-mediated class Acarbapenemase KPC-2 in clinical isolates of Klebsiella pneumoniae from South America. Antimicrob Agents Chemother. 2006 Aug;50(8):2880-2.

5. Doyle D, Peirano G, Lascols C, Lloyd T, Church DL, Pitout JDD. Laboratory detection of Enterobacteriaceae that produce carbapenemases. J Clin Microbiol. 2012 Dec;50(12):3877-80.

6. Schwaber MJ, Klarfeld-Lidji S, Navon-Venezia S, Schwartz D, Leavitt A, Carmeli Y. Predictors of carbapenem-resistant Klebsiella pneumoniae acquisition among hospitalized adults and effect of acquisition on mortality. Antimicrob Agents Chemother. 2008 Mar;52(3):1028-33.

7. Gasink LB, Edelstein PH, Lautenbach E, Synnestvedt M, Fishman NO. Risk factors and clinical impact of Klebsiella pneumoniaecarbapenemase-producing K. pneumoniae. Infect Control Hosp Epidemiol. 2009 Dec;30(12):1180-5.

8. Kwon S, Schweizer ML, Perencevich EN. National Institute of Allergy and Infectious Disease (NIAID) Funding for Studies of Hospital-Associated Bacterial Pathogens: Are Funds Proportionate to Burden of Disease? Antimicrob Resist Infect Control. 2012;1(1):5.

9. Neidell MJ, Cohen B, Furuya Y, Hill J, Jeon CY, Glied S, et al. Costs of healthcare- and community-associated infections with antimicrobial-resistant versus antimicrobial-susceptible organisms. Clin Infect Dis. 2012 Sep;55(6):807-15.

10. Correa L, Martino MDV, Siqueira I, Pasternak J, Gales AC, Silva CV, et al. A hospital-based matched case-control study to identify clinical outcome and risk factors associated with carbapenem-resistant Klebsiella pneumoniae infection. BMC Infect Dis. 2013;13:80.

11. María Eugenia Niño Mantilla. Diseño y validación de una escala basada en el concepto PIRO, una aproximación empírica a un modelo de pronóstico de los pacientes con sepsis. 2016. 18(2):214-8. 